\title{
gु
}

\section{Nonlinear Interaction between Single Photons}

\author{
T. Guerreiro, ${ }^{1}$ A. Martin, ${ }^{1}$ B. Sanguinetti, ${ }^{1}$ J. S. Pelc, ${ }^{2}$ C. Langrock,${ }^{2}$ M. M. Fejer, ${ }^{2}$ N. Gisin, ${ }^{1}$ H. Zbinden, ${ }^{1}$ \\ N. Sangouard, ${ }^{1, \dagger}$ and R. T. Thew ${ }^{1, *}$ \\ ${ }^{1}$ Group of Applied Physics, University of Geneva, 1211 Geneva 4, Switzerland \\ ${ }^{2}$ E.L. Ginzton Laboratory, Stanford University, 348 Via Pueblo Mall, Stanford, California 94305, USA
}

(Received 27 May 2014; published 22 October 2014)

\begin{abstract}
Harnessing nonlinearities strong enough to allow single photons to interact with one another is not only a fascinating challenge but also central to numerous advanced applications in quantum information science. Here we report the nonlinear interaction between two single photons. Each photon is generated in independent parametric down-conversion sources. They are subsequently combined in a nonlinear waveguide where they are converted into a single photon of higher energy by the process of sumfrequency generation. Our approach results in the direct generation of photon triplets. More generally, it highlights the potential for quantum nonlinear optics with integrated devices and, as the photons are at telecom wavelengths, it opens the way towards novel applications in quantum communication such as device-independent quantum key distribution.
\end{abstract}

DOI: 10.1103/PhysRevLett.113.173601

PACS numbers: 42.50.Dv, 03.67.Hk, 42.65.Ky

Observing nonlinear processes down to the quantum regime is a long sought after goal for quantum information science [1] as well as a fascinating concept in terms of fundamental physics, first being raised in the seminal work of Heisenberg and Euler [2]. It is only in recent years that materials and technologies have advanced to the point where one can probe this quantum nonlinear domain, and a few implementations have led to experimental realizations with attenuated classical laser light. This has included cross-phase modulation with weak classical light in atomic ensembles [3,4] and optical fibers [5], converting incident laser light into a nonclassical stream of photons [6,7] or Rydberg blockades [8] as well as all-optical switches with attenuated classical light in various atomic systems [9-15]. These atomic systems naturally operate with very narrow bandwidths and at specific wavelengths, typically in the visible regime. A grand challenge is to realize photonphoton interactions in materials that are less restrictive in terms of bandwidths and wavelengths. Of particular interest are photons at telecommunication wavelengths, as these provide the wiring, the flying qubits, for myriad applications in quantum communication [16,17]. A further challenge is to realize photon-photon interactions in a material that is not only less restrictive in terms of bandwidth, but also operating at room temperature.

Here we take an approach that exploits a parametric process, sum-frequency generation (SFG), in a nonlinear crystal [18-23]. The efficiency of nonlinear optical materials is constantly increasing and, by taking advantage of their inherently large bandwidth, one can work with pulsed systems and at higher repetition rates than with atomic systems. Important experimental results have been obtained in the context of quantum nonlinear optics with parametric processes [24-27], including the sum-frequency generation from two weak coherent states and between a coherent state and a true single photon. Here we take the next step and report, for the first time, a nonlinear interaction between two true independent single photons (Fock states), at telecom wavelengths, via SFG. Note that this experiment cannot be seen simply as the time reversal process of the nonlinear interaction presented in Refs. [24,25], nor analogue to that of Ref. [20], as spontaneous parametric down-conversion (SPDC) typically generates photon pairs correlated in spectra, while our photons were generated in independent sources and therefore have uncorrelated spectra. Our approach opens up perspectives unreachable with classical light. As an example, it allows one to generate photon triplets directly, as depicted in Fig. 1. One photon from each of two independent photon pair sources—-based

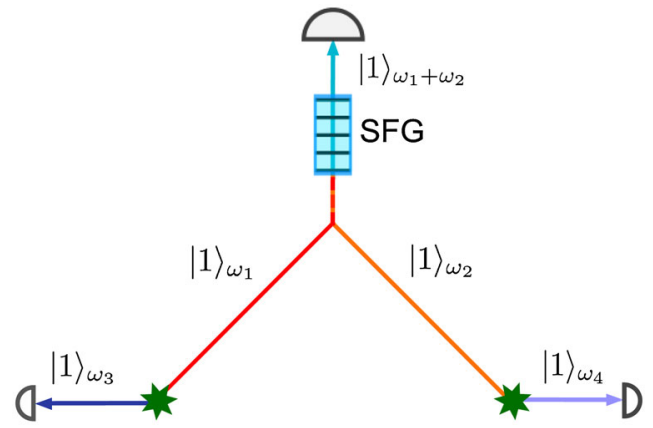

FIG. 1 (color online). Concept: Two single photons from independent photon pair sources are sent to a medium with $\chi^{(2)}$ nonlinearity and interact, generating a third photon carrying the sum of the energies and momenta of the input fields via SFG. As the photons sent to the SFG converter are at telecom wavelengths, the resulting photon triplet state allows, for example, the converted photon to herald the entanglement of the remaining two photon state at a distance. 
on SPDC-is sent to a nonlinear crystal where the two photons undergo SFG to generate a third photon. As the SFG requires one photon from each pair, the original pairwise correlation for each SPDC source is now mapped to the remaining three photon state- the SFG converted photon can herald the presence of the remaining two photons at distributed locations. This approach offers unique opportunities, for example, to herald entangled photon pairs - even remotely - and to perform quantum key distribution where the security is independent of the internal workings of the devices used to generate the secret key [28].

The experiment has three distinct parts: the generation of entangled photon pairs from independent SPDC sources [we can also interpret this as generating two independent single photons by two heralded single photon sources (HSPS)]; the parametric interaction of two single photons in a nonlinear waveguide; and, finally, the detection of the resulting single photon of higher energy in coincidence with the two remaining photons- the photon triplet. A schematic of the setup is shown in Fig. 2.

The single photons are generated via SPDC in two independent sources. HSPS1 and HSPS2 generate pairs at $807-1560 \mathrm{~nm}$ and $810-1551 \mathrm{~nm}$, respectively. By ensuring that the probability of creating a single pair in each source is much smaller than one, the detection of the visible (807, $810 \mathrm{~nm}$ ) photons heralds the creation of two independent single telecom wavelength $(1560,1551 \mathrm{~nm})$ photons. All of the photons are coupled into single-mode fibers with efficiencies $\sim 50 \%$. In principle this could be increased close to unity but is limited here by available pump power, which enforces a compromise in coupling geometry [30]. Importantly, the heralding photons are filtered such that the bandwidth of telecom photons is matched to the acceptance bandwidth of the SFG process; cf. below. To verify the single photon nature of these sources, we measured the conditional second-order autocorrelation functions $g_{1}^{(2)}(0)=0.0300 \pm 0.0005$ and $g_{2}^{(2)}(0)=0.0360 \pm 0.0004$, for HSPS1 and HSPS2, respectively.In this configuration, errors due to multipair emission are negligible [31].

The two fiber coupled telecom photons are then directed to a $4.5 \mathrm{~cm}$ fiber-pigtailed periodically poled lithium niobate (PPLN) waveguide that is quasi-phase matched to perform the SFG process $1560 \mathrm{~nm}+1551 \mathrm{~nm} \rightarrow 778 \mathrm{~nm}$. Figure 3 shows the results of a classical measurement of the phase matching conditions of the waveguide. The diagonal ridge corresponds to the SFG process, while the horizontal and vertical ridges result from the second harmonic generation (SHG) of each independent field. Following [27] and taking into account the acceptance bandwidth of the waveguide and the bandwidth of the interacting photons, which are measured to be $0.27 \mathrm{~nm}$, we determine a system conversion efficiency of $1.56 \times 10^{-8}$. This is close to the independently measured value, found to be $(1.5 \pm 0.3) \times 10^{-8}$.

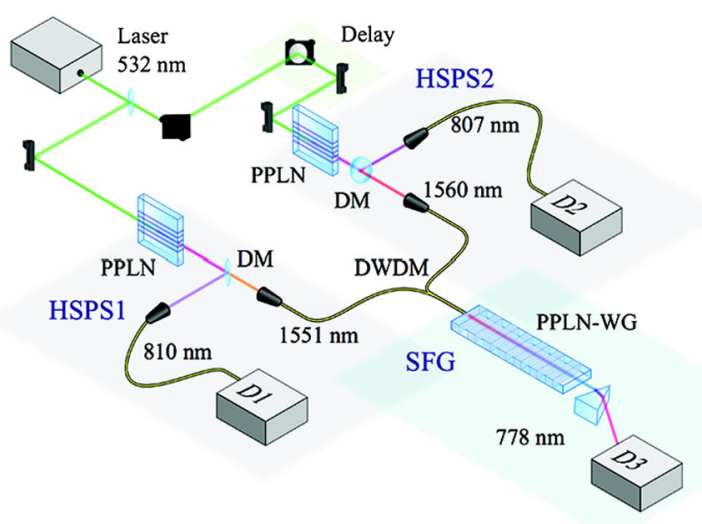

FIG. 2 (color online). Experimental setup. A mode-locked laser (Time-Bandwidth) generates $10 \mathrm{ps}$ pulses at $532 \mathrm{~nm}$ with a repetition rate of $430 \mathrm{MHz}$ and is used to pump the two heralded single photon sources (HSPS1 and HSPS2) based on PPLN nonlinear crystals. Each source receives an average pump power of $50 \mathrm{~mW}$. The generated photons are deterministically separated by dichroic mirrors (DM), collimated, and then collected into single-mode optical fibers. The pump light is extinguished by using high-pass filters with an extinction greater than $70 \mathrm{~dB}$ (Semrock), as well as a prism (not shown) before coupling into the optical fibers. Diffraction gratings (not shown) are employed to filter the heralding photons $(810,807 \mathrm{~nm})$ down to $\sim 0.3 \mathrm{~nm}$. In this configuration the telecom photons are projected onto a spectral mode that is matched to the acceptance bandwidth of the SFG process, which was measured to be $0.27 \mathrm{~nm}$. The two fiber coupled telecom photons are combined via a dense wavelength division multiplexer (DWDM) and directed to a $4.5 \mathrm{~cm}-$ long fiber-pigtailed type-0 PPLN waveguide [29]. Propagation loss in the waveguide is as small as $0.1 \mathrm{~dB} / \mathrm{cm}$. The unconverted photons are deterministically separated from the SFG photons by a prism (not shown) and the upconverted light is sent to a single photon detector $D 3$. The final photon triplet state is then detected by coincidence measurements between detectors $D 1, D 2$, and D3. The overall SFG conversion efficiency is $1.56 \times 10^{-8}$, including the coupling of the fiber pigtail, which is $70 \%$.

Finally, the detection scheme consists of three single photon detectors-D1, D2, and D3-based on Si avalanche photodiodes. Detector D3 (PicoQuant: $\tau$-SPAD-20) operates in free-running mode with an efficiency of $60 \%$ at $778 \mathrm{~nm}$ and a dark-count rate of $3.5 \mathrm{~s}^{-1}$. Detectors $D 1$ and D2 (Excelitas diodes with custom electronics) are gated (18 ns) devices with an efficiency of $60 \%$ and a dark-count probability of $10^{-6} / \mathrm{ns}$ [32]. All of the detection events from $D 1, D 2$, and $D 3$ are recorded by a time-to-digital converter.

Before running the nonlinear interaction measurement, it is necessary to make sure the interacting photons arrive at the same time into the nonlinear waveguide. To guarantee this, we seed HSPS2 with a continuous wave laser at $810 \mathrm{~nm}$, producing a pulsed coherent state at $1551 \mathrm{~nm}$ by difference frequency generation [33] to improve the signal to noise level. We record twofold coincidences between $D 3$ and $D 2$ as we scan the delay line placed before HSPS2. 


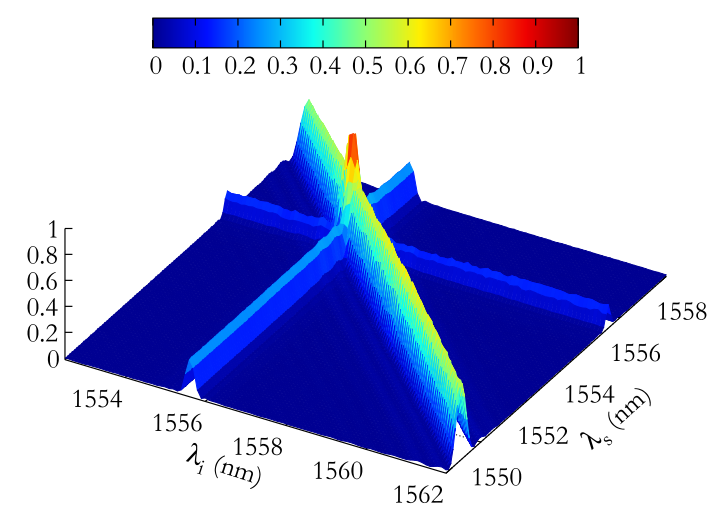

FIG. 3 (color online). Intensity plot of the classical SFG efficiency as a function of the wavelength of two input fields. The diagonal trace represents SFG between the input fields, while the horizontal and vertical traces signal the contributions from SHG of each independent input field. Note that the difference in height between the SFG and SHG signals is of about a factor of 4, as expected. It is also possible to see the oscillations from the $\operatorname{sinc}^{2}$ structure of the phase matching on the leading edge.

This allows for the temporal alignment of the interacting photons with picosecond resolution and also allows us to determine the exact position of the threefold coincidence peak in Fig. 4(a).

Once the two photons are temporally aligned, we can remove the seed laser and use the two sources in the heralded single photon configuration. We then proceed to record threefold coincidences between $D 1, D 2$, and $D 3$, where the appearance of a peak in the threefold detection time histogram signals the correlated generation of triplets of photons and hence that the interaction between the two independent telecom photons has taken place. We denote the delay between $D 1$ and $D 3$ as $\tau_{31}$, and the delay between $D 2$ and $D 3$ as $\tau_{32}$.

The threefold coincidences between $D 1, D 2$, and $D 3$ are shown in the time-of-arrival histogram in Fig. 4(a). Each bin of this histogram corresponds to an acquisition window of $2.3 \mathrm{~ns}$ for each detector matching the repetition rate of the pump laser. We integrate for 260 hours and observe a well-defined coincidence peak exactly where it is expected. Moreover, Fig. 4(b) shows the histogram of threefold coincidence counts. One sees a Poissonian distribution for the background noise with a mean value of 35 , calculated by averaging overall counts in the 100 bins of Fig. 4(a). The background noise is dominated by the detection of photons at $D 1$ and $D 2$ in coincidence with dark counts $\left(3.5 \mathrm{~s}^{-1}\right)$ from detector $D 3$ [27]. The three photon signature is the single bin containing 80 counts, which has a statistical significance of over 7 standard deviations with respect to the background. Furthermore, given the Poisson distribution with a mean value of 35 , the probability of having a pixel with 80 accidental counts is of the order of $10^{-11}$.

Our theoretical model of the system takes into account the source emission probability, the losses of the setup, the SFG conversion probability, and the detector efficiency and noise levels. We estimated a rate of 0.40 threefold coincidences versus a rate of 0.20 threefold noise events, per hour, while the observed values are 0.31 and 0.13 coincidences, respectively. The measured values are both slightly reduced due to source alignment drifting over the long integration time. Nonetheless, the signal to noise ratio is in good agreement with our predictions. (a)

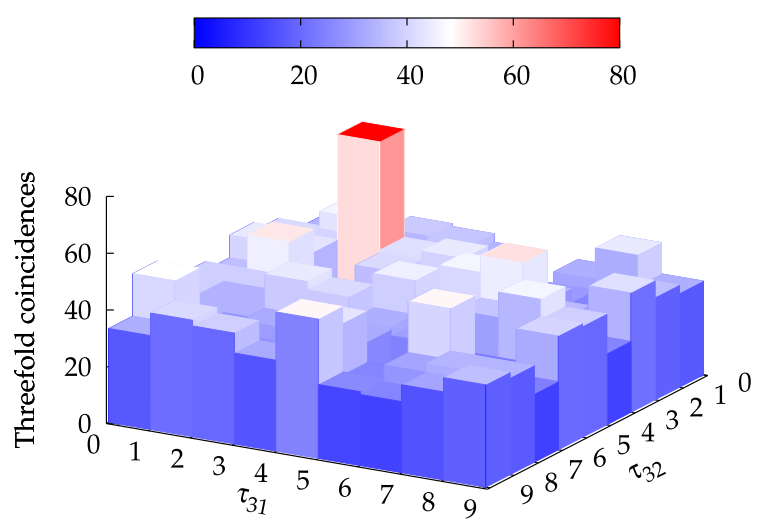

(b)

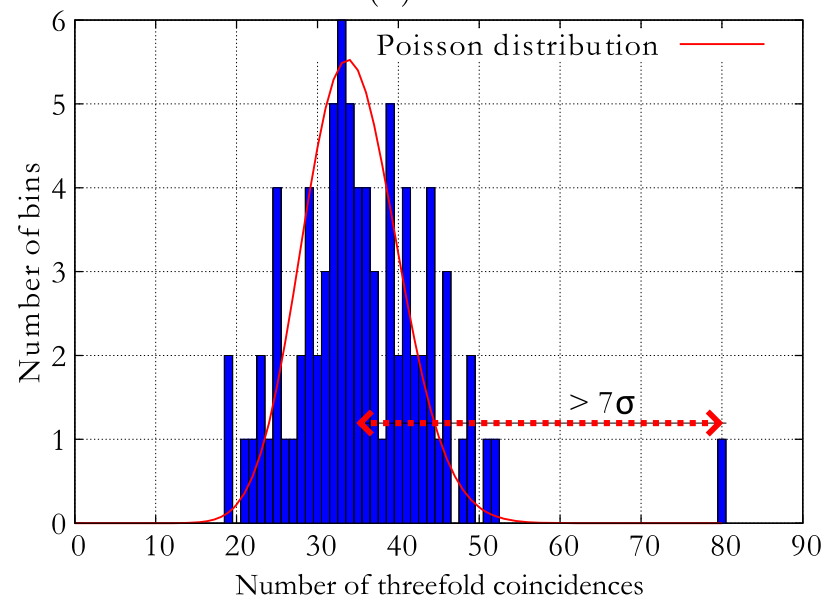

FIG. 4 (color online). (a) The central peak (red) emerging from the background noise in the time-of-arrival threefold coincidence histogram is a clear signature of the photon-photon interaction and the nonclassical correlations for the photon triplet state. The axis labeled $\tau_{31}$ shows the delay between detectors $D 1$ and $D 3$, while $\tau_{32}$ shows the delay between $D 2$ and $D 3$. Each pixel is composed of $2.3 \mathrm{~ns}$ bins, defined by the laser repetition rate. (b) The histogram of threefold coincidence counts shows a Poissonian distribution for the noise with a mean value of 35 . The single peak at 80 corresponds to the true threefold coincidences and exceeds the mean value by over 7 standard deviations $(7 \sigma)$. 
Despite the challenging nature of the current experiment, higher nonlinear conversion efficiencies allowed us to go from interactions between two coherent states [28] to interacting a single photon and a weak coherent state [27]. Further improvements for the photon pair sources [30] and detectors [32] have now made it possible to demonstrate the first nonlinear interaction between two independent quantum systems. The nonlinearity reported here already offers promising perspectives-for example, the implementation of quantum key distribution-where the secrecy is independent of the internal workings of the devices that are used to create the key (device-independent quantum key distribution). An important aspect of this framework is that the combination of entangled photon pairs at telecom wavelengths and the SFG process allows for maximally entangled photon pairs to be created at a distance while being heralded through the detection of converted photons [28]. The presented demonstration of photon triplets by photon-photon interaction is also promising from a fundamental perspective: for instance, unambiguously excluding local hidden variable models of entanglement in a loophole-free Bell-type experiment [34,35], opening the way for investigating novel quantum correlations [36], and providing a platform for studying exotic states of light and quantum optical solitons [37].

Looking further ahead, higher efficiency nonlinear interactions could be obtained by using tight spatial confinement of the optical modes [38], from the use of highly nonlinear organic materials [39], or by exploiting weak measurements based on pre- and postselected states, as pointed out in Ref. [40]. In addition to these exciting perspectives, we believe that our demonstration of an interaction between two independent single photons will strongly stimulate research in nonlinear optics in the quantum regime.

The authors would like to thank E. Pomarico for the useful discussion. This Letter was partially supported by the EU projects SIQS and CHIST-ERA: QScale and DIQIP, as well as the Swiss NCCR QSIT, the Swiss National Foundation SNSF (Grant No. PP00P2-150579) and the U.S. AFOSR (Grant No. FA9550-12-1-0110).

*robert.thew@unige.ch; http://www.unige.ch/gap/optics

"Present address: Department of Physics, University of Basel CH-4056, Basel, Switzerland.

[1] M. A. Nielsen and L. I. Chuang, Quantum Computation and Quantum Information, Cambridge Series on Information and the Natural Sciences (Cambridge University Press, Cambridge, England, 2000).

[2] W. Heisenberg and H. Euler, Z. Phys. 98, 714 (1936).

[3] H. Kang and Y. Zhu, Phys. Rev. Lett. 91, 093601 (2003).

[4] Y. F. Chen, C. Y. Wang, S. H. Wang, and I. A. Yu, Phys. Rev. Lett. 96, 043603 (2006).
[5] N. Matsuda, R. Shimizu, Y. Mitsumori, H. Kosaka, and K. Edamatsu, Nat. Photonics 3, 95 (2009).

[6] K. M. Birnbaum, A. Boca, R. Miller, A. D. Boozer, T. E. Northup, and H. J. Kimble, Nature (London) 436, 87 (2005).

[7] A. Kubanek, A. Ourjoumtsev, I. Schuster, M. Koch, P. Pinkse, K. Murr, and G. Rempe., Phys. Rev. Lett. 101, 203602 (2008).

[8] T. Peyronel, O. Firstenberg, Q.-Y. Liang, S. Hofferberth, A. V. Gorshkov, T. Pohl, M. D. Lukin, and V. Vuletić, Nature (London) 488, 57 (2012).

[9] R. J. Thompson, G. Rempe, and H. J. Kimble, Phys. Rev. Lett. 68, 1132 (1992).

[10] M. Brune, F. Schmidt-Kaler, A. Maali, J. Dreyer, E. Hagley, J. Raimond, and S. Haroche, Phys. Rev. Lett. 76, 1800 (1996).

[11] A. M. C. Dawes, L. Illing, S. M. Clark, and D. J. Gauthier, Science 308, 672 (2005).

[12] J. Hwang, M. Pototschnig, R. Lettow, G. Zumofen, A. Renn, S. Götzinger, and V. Sandoghdar., Nature (London) 460, 76 (2009).

[13] D. Englund, A. Majumdar, M. Bajcsy, A. Faraon, P. Petroff, and J. Vučković, Phys. Rev. Lett. 108, 093604 (2012).

[14] T. Volz, M. Pototschnig, R. Lettow, G. Zumofen, A. Renn, S. Götzinger, and V. Sandoghdar, Nature (London) 460, 76 (2009).

[15] W. Chen, K. M. Beck, R. Bucker, M. Gullans, M. D. Lukin, H. Tanji-Suzuki, and V. Vuletic, Science 341, 768 (2013).

[16] N. Gisin and R. T. Thew, Nat. Photonics 1, 165 (2007).

[17] N. Sangouard and H. Zbinden, J. Mod. Opt. 59, 1458 (2012).

[18] S. Tanzilli et al., Laser Photonics Rev. 6, 115 (2012).

[19] Y. H. Kim, S. P. Kulik, and Y. Shih, Phys. Rev. Lett. 86, 1370 (2001).

[20] B. Dayan, A. Peer, A. A. Friesem, and Y. Silberberg, Phys. Rev. Lett. 94, 043602 (2005).

[21] G. Giorgi, P. Mataloni, and F. De Martini, Phys. Rev. Lett. 90, 027902 (2003).

[22] A. P. Vandevender and P. G. Kwiat, J. Mod. Opt. 51, 1433 (2004).

[23] R. T. Thew, H. Zbinden, and N. Gisin, Appl. Phys. Lett. 93, 071104 (2008).

[24] H. Hübel, D. R. Hamel, A. Fedrizzi, S. Ramelow, K. J. Resch, and T. Jennewein, Nature (London) 466, 601 (2010).

[25] L. K. Shalm, D. R. Hamel, Z. Yan, C. Simon, K. J. Resch, and T. Jennewein, Nat. Phys. 9, 19 (2013).

[26] N. K. Langford, S. Ramelow, R. Prevedel, W. J. Munro, G. J. Milburn, and A. Zeilinger., Nature (London) 478, 360 (2011).

[27] T. Guerreiro, E. Pomarico, B. Sanguinetti, N. Sangouard, J. S. Pelc, C. Langrock, M. M. Fejer, H. Zbinden, R. T. Thew, and N. Gisin, Nat. Commun. 4, 2324 (2013).

[28] N. Sangouard, B. Sanguinetti, N. Curtz, N. Gisin, R. Thew, and H. Zbinden., Phys. Rev. Lett. 106, 120403 (2011).

[29] K. R. Parameswaran, R. K. Route, J. R. Kurz, R. V. Roussev, M. M. Fejer, and M. Fujimura, Opt. Lett. 27, 179 (2002).

[30] T. Guerreiro, A. Martin, B. Sanguinetti, N. Bruno, H. Zbinden, and R. T. Thew, Opt. Express 21, 27641 (2013). 
[31] P. Sekatski, N. Sangouard, F. Bussières, C. Clausen, N. Gisin, and H. Zbinden, J. Phys. B 45, 124016 (2012).

[32] T. Lunghi, E. Pomarico, C. Barreiro, D. Stucki, B. Sanguinetti, and H. Zbinden, Appl. Opt. 51, 8455 (2012).

[33] N. Bruno, A. Martin, and R. T. Thew, Opt. Commun. 327, 17 (2014).

[34] A. Cabello and F. Sciarrino, Phys. Rev. X 2, 021010 (2012).

[35] V. Caprara Vivoli et al.., arXiv:1405.1939.

[36] K. Banaszek and P. L. Knight, Phys. Rev. A 55, 2368 (1997).
[37] K. V. Kheruntsyan and P. D. Drummond, Phys. Rev. A 58, 2488 (1998).

[38] S. Kurimura, Y. Kato, M. Maruyama, Y. Usui, and H. Nakajima, Appl. Phys. Lett. 89, 191123 (2006).

[39] M. Jazbinsek, L. Mutter, and P. Gunter, IEEE J. Sel. Top. Quantum Electron. 14, 1298 (2008).

[40] A. Feizpour, X. Xing, and A. M. Steinberg, Phys. Rev. Lett. 107, 133603 (2011).

[41] See Supplemental Material at http://link.aps.org/ supplemental/10.1103/PhysRevLett.113.173601 for details about the calculation of the expected rates. 